\title{
Efecto de la estructuración por macrófitas y por recursos alimentarios en la distribución horizontal de tecamebas y rotíferos en un lago andino patagónico
}

\author{
Effect of macrophytes and food resources on the horizontal distribution of testate amoebae \\ and rotifers in an Andean-Patagonian lake
}

MARCELA BASTIDAS-NAVARRO \& BEATRIZ MODENUTTI

\begin{abstract}
Laboratorio de Limnología, Centro Regional Universitario Bariloche, CONICET-Universidad Nacional del Comahue, Quintral 1250, 8400 Bariloche, Argentina

*e-mail para correspondencia: bastidas@crub.uncoma.edu.ar
\end{abstract}

\begin{abstract}
RESUMEN
La presencia de macrófitas en la zona litoral lacustre trae aparejada condiciones diferentes con respecto a la zona pelágica, en particular en la disponibilidad de recursos. Por esta razón, las zonas litorales pueden presentar una mayor biodiversidad. En este trabajo se analizó la distribución horizontal de tecamebas y rotíferos en el lago Escondido (Argentina), vinculándola con los recursos alimentarios presentes en las diferentes zonas. Se tomaron muestras para el estudio del fitoplancton y zooplancton durante verano y primavera (2001-2003) en cuatro estaciones litorales y una pelágica. Se realizó el recuento de rotíferos y tecamebas y se determinó la abundancia y la biomasa fitoplanctónica. Los recursos alimentarios fueron clasificados en nanoplancton $(<20 \mu \mathrm{m})$ y fitoplancton de red $(>20 \mu \mathrm{m})$. El nanoplancton estuvo compuesto por nanoflagelados y el fitoplancton de red por diatomeas, cianófitas y clorófitas. La biomasa de estas fracciones varió significativamente, observándose una predominancia del nanoplancton en la zona pelágica y del fitoplancton de red en la zona litoral. Los mayores valores de riqueza y de diversidad de tecamebas y rotíferos fueron observados en las estaciones litorales, no hallándose diferencias significativas entre las zonas con diferentes macrófitas. El análisis de ACC evidenció cuatro grupos. Por un lado, Keratella cochlearis, Synchaeta spp., Polyarthra vulgaris y Collotheca mutabilis, asociadas a las muestras pelágicas, se relacionaron con una alta abundancia de nanoplancton. Por otro lado, los rotíferos Trichocerca spp., Lecane spp. y Euchlanis spp., y las tecamebas Difflugia pyriformis y Trinema enchelys se relacionaron con el fitoplancton de red y las muestras litorales. Además, un tercer grupo reunió a especies (Lecane spp. y Arcella spp.) presentes en ambas zonas y en relación con una baja abundancia del fitoplancton de red. Por último, el rotífero Synchaeta spp. y las muestras pelágicas de primavera se relacionaron con el aumento de la abundancia de la cianofita Coelosphaerium kuetzingianum. Las diferencias señaladas indican que los recursos alimentarios serían un factor determinante en la distribución de especies de tecamebas y rotíferos en el lago Escondido. Para estos zoopláncteres de pequeño tamaño las macrófitas litorales brindarían alimento al favorecer el incremento del fitoplancton de red.
\end{abstract}

Palabras clave: zona litoral, zona pelágica, tecamebas, rotíferos, recursos algales.

\begin{abstract}
The presence of macrophytes in the littoral zone of lakes produces particular conditions including higher resource availability for consumers. For this reason, the littoral zone is generally the area with the highest diversity of lakes and rivers. In this work we studied the horizontal distribution of testate amoebae and rotifers in Lago Escondido (Argentina) in relation to food resources availability. The study was carried out along a north-south transect that includes the littoral and the pelagic zone of the lake. Phytoplankton and zooplankton were sampled during summer and spring (2001-2003) in five sampling stations: one pelagic and four littoral. Rotifers and testate amoebae, as well as phytoplanktonic algae abundance and biomass were estimated. Food resources were classified as nanoplankton $(<20 \mu \mathrm{m})$ and net phytoplankton $(>20 \mu \mathrm{m})$ and the biovolume of these fractions varied significantly within the pelagic and littoral zones of the lake. Nanoplankon dominated the pelagic zone and was mainly composed by nanoflagellates while net phytoplankton prevailed in the littoral zone and was composed by diatoms, cyanophytes and chlorophytes. The highest number of species and diversity of testate amoebae and rotifers were observed in the littoral stations; nevertheless, no significant differences between the zones colonized by different macrophytes were
\end{abstract}


observed. The CCA analysis showed four different groups. Keratella cochlearis, Synchaeta spp., Polyarthra vulgaris and Collotheca mutabilis characterized the pelagic samples and were related with a high abundance of nanoplankton. On the other hand, rotifers like Trichocerca spp., Lecane spp. and Euchlanis spp. and the testate amoebae Difflugia pyriformis and Trinema enchelys associated with the littoral samples were related with high abundances of net phytoplankton. Besides, a third group of species (Lecane spp. and Arcella spp.), presented in both zones, was mainly related with a decrease in abundance of net phytoplankton. Finally, the rotifer Synchaeta spp. was associated with the spring pelagic samples when the cyanophyte Coelosphaerium kuetzingianum increased its abundance. Summarizing, in Lago Escondido, food resources could be an important factor driving testate amoeba and rotifer distribution. Therefore the major role of macrophytes for this small-bodied zooplankton is food supply through an increment of net phytoplankton.

Key words: littoral zone, pelagic zone, testate amoebae, rotifers, algal resources.

\section{INTRODUCCIÓN}

La presencia de macrófitas en la zona litoral de un lago trae aparejada una serie de diferencias notables, con respecto a la zona de aguas abiertas. Entre ellas, se destacan una variabilidad espacial y temporal de la química del agua, oxígeno, pH y temperatura como resultado de la actividad fotosintética, de la descomposición y la menor mezcla del agua (Vitt \& Bayley 1984, Cyr \& Downing 1988a, 1988b). Las macrófitas son reconocidas por ser uno de los principales factores que influyen sobre la estructura espacial de microcrustáceos y de la macrofauna en el litoral de los lagos (Dvorak 1970, Shiel 1976, Talbot \& Ward 1987). Esto se debe a que las macrófitas y la red de algas que se establece sobre las superficies de las plantas provee al zooplancton de intersticios donde refugiarse de los predadores, constituye una importante fuente de alimentación y también brinda un sustrato para desovar (Dvorak 1970, Dvorak \& Best 1982, Rooke 1984, 1986a, 1984b, Diehl 1992). Por estas razones, la zona litoral es a menudo la región con mayor diversidad zooplanctónica de lagos y ríos (Duggan 2001), ya que en ella coexisten organismos vinculados a la columna de agua así como también aquellos asociados a las macrófitas que pueden soltarse y desplazarse al agua circundante (Schweizer 1997, Duggan 2001).

En las zonas litoral y pelágica de los lagos, los tecamebianos y los rotíferos son consumidores de gran importancia. Las tecamebas pueden incluso ser los organismos litorales más abundantes y contribuir significativamente a la productividad del ambiente (Lansac-Tôha et al. 1997, Alekperov \& Snegovaya 2000, Bini et al. 2003). Las tecamebas se alimentan principalmente de partículas grandes $(\geq 100 \mu \mathrm{m})$ incluyendo materia orgánica particulada, algas y protistas, pequeños metazoos y contenidos celulares de células de macrófitas (Gilbert et al. 2000), que obtienen mediante la emisión de pseudópodos. Por otra parte, en hábitats litorales con macrófitas también se ha citado una mayor diversidad y abundancia de rotíferos (Nogrady et al. 1993, Pejler 1995, Duggan 2001). Los rotíferos son de gran importancia como consumidores primarios del ecosistema lacustre (Nogrady et al. 1993). La estructura del aparato masticador de estos organismos condiciona su dieta. Las especies filtradoras presentan mástax ramado, maleado o malleorramado, ingiriendo un rango de partículas entre 3 a $17 \mu \mathrm{m}$ (Nogrady et al. 1993). Por otro lado, las especies predadoras y raptoras presentan mástax virgado, uncinado o incudado, cuya estructura les permite acceder a partículas de mayor tamaño (> $20 \mu \mathrm{m})$, incluyendo tanto presas móviles (protozoos y rotíferos) como algas de mayor tamaño celular (dinoflagelados, diatomeas) (Stemberger \& Gilbert 1987, Nogrady et al. 1993).

Los productores bénticos de los lagos andino-patagónicos incluyen a macrófitas y perifiton, cuyo desarrollo varía de acuerdo a la ubicación y la morfología de la cubeta. En la región andina hay numerosos lagos pequeños y someros con desarrollo de una zona litoral vegetada (Perotti et al. 2005). A pesar que existen numerosas contribuciones sobre la dinámica de rotíferos y otro microzooplancton en la zona pelágica de lagos andinos someros y profundos (Balseiro \& Modenutti 1990, Schmid-Araya 1991, 1993, Modenutti 1994, Modenutti \& Diéguez 1996, Diéguez et al. 1998, Modenutti et al. 2000, Modenutti et al. 2005, entre otros), las zonas litorales de estos ambientes están prácticamente sin investigar. 
Considerando la falta de estudios en esta región lacustre de los lagos andinos y que estas pueden poseer una particular importancia para el ecosistema, resulta importante el análisis de su papel en la dinámica lacustre. En este estudio se analizó la distribución horizontal de tecamebas y rotíferos en un lago andino argentino somero. También se analizaron las probables vinculaciones entre las zonas, mediante su caracterización a partir de especies comunes y de distribución restringida. Como hipótesis inicial se planteó que la distribución de los recursos alimentarios algales y de las macrófitas presentes en las diferentes zonas determina la distribución de tecamebas y rotíferos, esperándose encontrar diferencias en la abundancia, diversidad y composición específica de los grupos estudiados.

\section{MATERIALES Y MÉTODOS}

El lago Escondido es un pequeño lago somero perteneciente a la cuenca del lago Nahuel Huapi. Se ubica a los $41^{\circ} 03$ '29' 'S y $71^{\circ} 34^{\prime} 20^{\prime \prime}$ 'O a 764 m sobre el nivel del mar, en la Provincia de Río Negro, Argentina. Presenta una profundidad máxima de $8 \mathrm{~m}$ y un área de $8 \mathrm{ha}$. La zona litoral se encuentra colonizada por la macrófita palustre Schoenoplectus californicus (Meyer) Soják (= Scirpus californicus) y la sumergida Potamogeton linguatus Hangström. La primera se dispone desde la línea de costa hasta los $2 \mathrm{~m}$ de profundidad y la segunda se ubica en forma continua entre los $2 \mathrm{~m}$ hasta $\operatorname{los} 5 \mathrm{~m}$ de profundidad aproximadamente (Fig. 1). El comportamiento térmico fue descripto como monomíctico cálido, con períodos de mezcla durante otoño e invierno (Balseiro \& Modenutti 1990). Díaz \& Pedrozo (1993) lo describen como un lago oligotrófico de aguas muy diluidas dominadas por bicarbonato de calcio y silicatos disueltos y con bajo contenido de clorofila $a(1,8$ $\mathrm{mg} \mathrm{m}^{-3}$ en verano). La presencia de macrófitas en la zona litoral, las epífitas asociadas y el bosque circundante contribuyen a una alta concentración de carbono orgánico disuelto (COD 2,66 $\mathrm{mg} \mathrm{L}^{-1}$ ) en este lago con respecto a los lagos profundos del área (Morris et al. 1995). La estructura y dinámica del fitoplancton fueron analizadas por Díaz y Pedrozo (1993) y el zooplancton por Balseiro \& Modenutti (1990) y Modenutti \& Diéguez (1996).
Se realizaron muestreos en diciembre de 2001 , enero y noviembre de 2002 y febrero de 2003. En cada muestreo se establecieron cinco estaciones de muestreo a lo largo de una transecta con sentido norte-sur (Fig. 1). Dos de las estaciones se situaron en la zona colonizada por S. californicus (E1 y E5), tomándose muestras en la superficie y a $1 \mathrm{~m}$ de profundidad. Otras dos estaciones se ubicaron en la zona colonizada por $P$. linguatus (E2 y E4), donde se muestreó en la superficie y a los $3 \mathrm{~m}$. Una quinta estación se situó en la zona pelágica (E3), muestreándose en superficie, a los 3 y los 6 m de profundidad. En cada ocasión de muestreo se registró la temperatura y el oxígeno disuelto con un termistor y un oxímetro (YSI 59), respectivamente. Se tomaron muestras con una trampa SchindlerPatalas de $12 \mathrm{~L}$, tanto en la columna de agua como en el agua circundante a las macrófitas. El volumen de agua obtenido fue trasladado inmediatamente después de la colecta al laboratorio bajo condiciones de oscuridad para los análisis posteriores. Un litro de agua fue destinado para la determinación de clorofila $a$ y concentración de COD. Estas determinaciones se llevaron a cabo dentro de la media hora posterior a la colecta de las muestras. Un volumen de $250 \mathrm{~mL}$ se fijó con lugol acético (1 $\%)$ para el recuento algal. Finalmente, $10 \mathrm{~L}$ fueron filtrados con red de plancton de $55 \mu \mathrm{m}$ y fijados con formol (4\%) para el estudio del zooplancton. Cabe señalar que no se tomaron muestras en la E5 en el mes de noviembre.

En el laboratorio, la concentración de COD se estimó espectrofotométricamente en agua filtrada (GF/F) ajustándose los valores registrados a la regresión de Morris et al. (1995). La biomasa algal se estimó a través de la determinación de la concentración de clorofila $a$ siguiendo la metodología de Nusch (1980). La medición se efectuó filtrando 250 $\mathrm{mL}$ de agua a través de filtros GF/F. El recuento algal se realizó siguiendo el método de Utermöhl con microscopio invertido en cámaras de $50 \mathrm{~mL}$. Los individuos fueron medidos en cada caso y clasificados, de acuerdo a su dimensión axial máxima, en nanoplancton $(<20 \mu \mathrm{m})$ y fitoplancton de red (> $20 \mu \mathrm{m}$ ). También se calcularon los biovolúmenes correspondientes a estas fracciones, aproximando la forma celular a formas geométricas o a una adecuada 
combinación de ellas (Sun \& Liu 2003). El recuento de rotíferos y tecamebas se realizó en cámara de Sedgwick-Rafter de $1 \mathrm{~mL}$ bajo microscopio directo. En el caso particular de las tecamebas, para no obviar especies menores de $55 \mu \mathrm{m}$, también se observaron y contaron las muestras de botella sin filtrar fijadas con lugol bajo microscopio invertido.

La diversidad específica de tecamebas y rotíferos fue calculada en cada estación de muestreo para cada profundidad mediante el índice de diversidad de Shannon-Weaver (H) (Southwood 1978). Las variaciones en el biovolumen del nanoplancton y del fitoplancton de red, en la abundancia del fitoplancton, en la abundancia de rotíferos y en la diversidad de tecamebas y rotíferos fueron analizadas mediante un ANOVA de dos vías y luego una prueba de comparaciones múltiples de StudentNewman-Keuls en los casos necesarios (Underwood 1997). Previamente se verificó que se cumplan los requisitos de normalidad y homocedasticidad. Cuando fue requerido, los datos se transformaron con función logarítmica. Los patrones de asociación entre la abundancia de tecamebas y rotíferos y la abundancia de nanoplancton y fitoplancton de red (variables del recurso alimentario), fueron analizados con el método de ordenación de Análisis de Correspondencia Canónica (ACC) (Leps \& Smilauer 2003). Los cálculos fueron realizados con el programa CANOCO (Ter Braak \& Smilauer 2002). Los datos analizados correspondieron a valores de abundancia de especies de tecamebas y rotíferos presentes en las diferentes muestras. Se incluyeron en el análisis las especies que presentaron, por lo menos en una muestra, una frecuencia relativa mayor al $10 \%$. La significancia de la ordenación de los ejes fue determinada mediante el test de permutaciones de Monte Carlo.

\section{RESULTADOS}

En los muestreos realizados el lago Escondido presentó gradientes térmicos y estratificación en la columna de agua, con un máximo de 22,5 ${ }^{\circ} \mathrm{C}$ en la superficie y un mínimo de $9{ }^{\circ} \mathrm{C}$ a los 6 $\mathrm{m}$ de profundidad (Tabla 1). Las estaciones situadas en la zona de Potamogeton linguatus

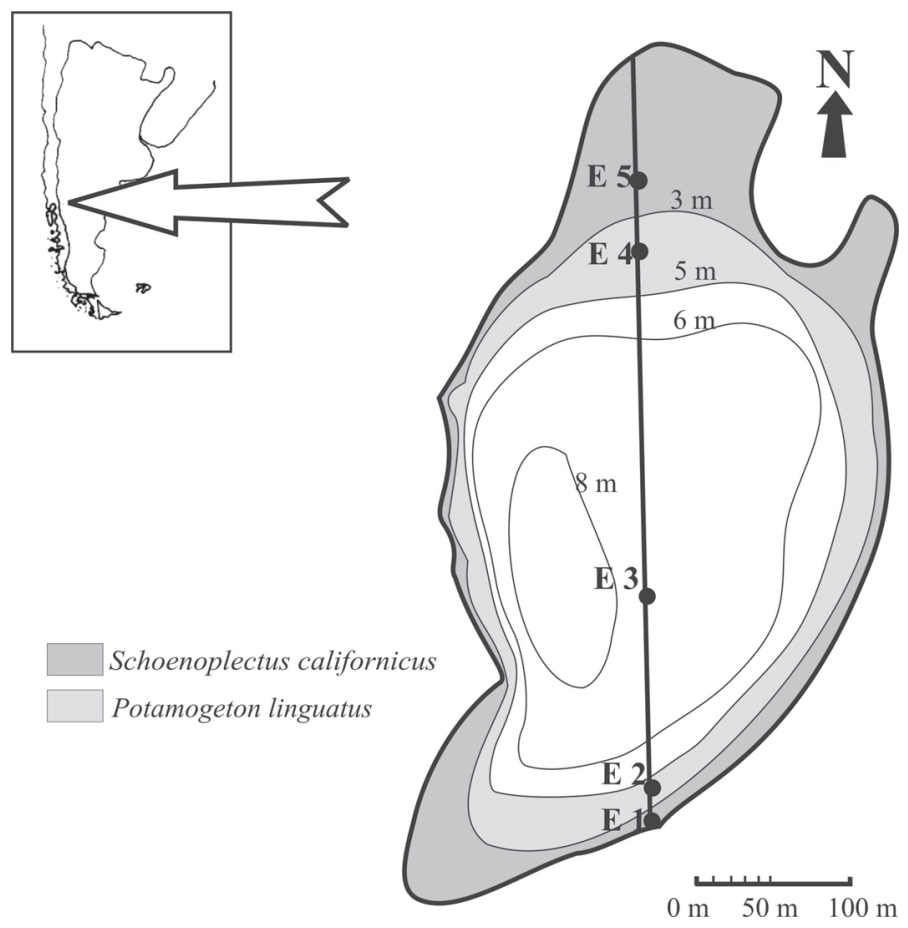

Fig. 1: Ubicación del Lago Escondido, transecta y estaciones de muestreo. Location of Lake Escondido, and sampling stations. 
(E2 y E4, Fig. 1) también mostraron gradientes, aunque menos marcados, ya que la diferencia de temperatura entre superficie y fondo nunca superó a los $5^{\circ} \mathrm{C}$. Las estaciones ubicadas en la zona de Schoenoplectus californicus (E1 y E5, Fig. 1) presentaron temperatura homogénea desde la superficie hasta el fondo. Por esta razón, aunque se tomaron muestras en superficie y a $1 \mathrm{~m}$ de profundidad en esta zona, en la Tabla 1 solo figuran los valores del nivel superficial, ya que en todas las ocasiones de muestreo los resultaron obtenidos entre $0 \mathrm{~m}$ y 1 $\mathrm{m}$ fueron coincidentes. Las estaciones litorales en la zona de S. californicus presentaron los valores máximos de temperatura con respecto al resto de las estaciones registrándose un máximo de $25,5^{\circ} \mathrm{C}$ en E1 (Tabla 1 ).

La concentración de oxígeno disuelto fue alta durante todo el período muestreado, observándose saturación de oxígeno en todas las estaciones de muestreo y en toda la columna de agua (Tabla 1). El lago exhibió valores de
COD comparativamente altos oscilando alrededor de los 4,5 $\mathrm{mg} \mathrm{C} \mathrm{L}^{-1}$. Los valores máximos fueron registrados en la zona litoral, alcanzando 5,5 $\mathrm{mg} \mathrm{C} \mathrm{L}^{-1}$ en la E1 colonizada por S. californicus. Por el contrario, en la zona colonizada por Potamogeton linguatus y en la zona pelágica se observaron valores menores de concentración (Tabla 1), llegando a un mínimo de 4,1 $\mathrm{mg} \mathrm{C} \mathrm{L}^{-1}$ en febrero. La concentración de clorofila a varió entre las diferentes zonas, registrándose los mayores valores en las muestras litorales (máximo de $12,9 \mathrm{mg} \mathrm{L}^{-1}$ ) y los menores, en la superficie de la estación pelágica (mínimo de $0,8 \mathrm{mg} \mathrm{L}^{-1}$ ).

Con respecto a la distribución del fitoplancton (Fig. 2), se observaron diferencias significativas en el biovolumen del nanoplancton entre las zonas del lago y entre las fechas de muestreo, sin observarse interacción entre ambos factores (Tabla 2). La zona pelágica difirió significativamente de las dos zonas colonizadas por macrófitas (prueba a

TABLA 1

Temperatura, concentración de oxígeno disuelto, COD (carbono orgánico disuelto) y clorofila $a$ en las diferentes zonas y niveles muestreados en el Lago Escondido. Los valores se indican como medias $\pm \mathrm{DE}$; entre paréntesis se presentan los valores mínimos y máximos

Temperature, dissolved oxygen concentration, DOC (dissolved organic carbon) and chlorophyll $a$ for the different zones and depths of Lake Escondido. Values are given as means \pm SD; maximum and minimum values are shown in brackets

\begin{tabular}{|c|c|c|c|c|}
\hline Parámetro & Profundidad & $\begin{array}{l}\text { Zona litoral } \\
\text { S. californicus } \\
\text { (E1 y E5) }\end{array}$ & $\begin{array}{l}\text { Zona litoral } P . \text { linguatus } \\
\text { (E2 y E4) }\end{array}$ & Zona pelágica (E3) \\
\hline \multirow[t]{3}{*}{ Temperatura $\left({ }^{\circ} \mathrm{C}\right)$} & $0 \mathrm{~m}$ & $22,7 \pm 1,4(21,5-25,5)$ & $21 \pm 2,3(16-23,5)$ & $20,2 \pm 2,8(17-22,5)$ \\
\hline & $3 \mathrm{~m}$ & - & $18,3 \pm 4,1(11-21)$ & $17,3 \pm 4,6(12-20,5)$ \\
\hline & $6 \mathrm{~m}$ & - & - & $11,8 \pm 2,6(9-14,2)$ \\
\hline \multirow[t]{3}{*}{ Oxígeno disuelto $\left(\mathrm{mg} \mathrm{L}^{-1}\right)$} & $0 \mathrm{~m}$ & $8,3 \pm 0,6(7,7-9,2)$ & $9,7 \pm 1,3(8,8-12)$ & $9,9 \pm 1(8,8-10,7)$ \\
\hline & $3 \mathrm{~m}$ & - & $8,5 \pm 1,4(6,5-10,5)$ & $9,1 \pm 1,7(7,7-11)$ \\
\hline & $6 \mathrm{~m}$ & - & - & $8,4 \pm 1,6(7,3-10,2)$ \\
\hline \multirow[t]{3}{*}{$\operatorname{COD}\left(\mathrm{mg} \mathrm{L}^{-1}\right)$} & $0 \mathrm{~m}$ & $4,7 \pm 0,7(4,2-5,5)$ & $4,4 \pm 0,4(4,1-4,9)$ & $4,5 \pm 0,5(4,1-4,8)$ \\
\hline & $3 \mathrm{~m}$ & - & $4,4 \pm 0,4(4,1-4,9)$ & $4,5 \pm 0,6(4,1-4,9)$ \\
\hline & $6 \mathrm{~m}$ & - & - & $4,5 \pm 0,4(4,2-4,8)$ \\
\hline \multirow[t]{3}{*}{ Clorofila $a\left(\mu \mathrm{g} \mathrm{L}^{-1}\right)$} & $0 \mathrm{~m}$ & $2,5 \pm 1,1(1,6-3,7)$ & $1,3 \pm 0,6(0,9-1,7)$ & $0,9 \pm 0,1(0,8-1,01)$ \\
\hline & $3 \mathrm{~m}$ & - & $8,7 \pm 3,6(6,1-12,9)$ & $1,3 \pm 0,2(1,1-1,4)$ \\
\hline & $6 \mathrm{~m}$ & - & - & $2,4 \pm 0,(1,8-2,92)$ \\
\hline
\end{tabular}


posteriori de Student-Newman-Keuls, pelágica versus $P$. linguatus, $\mathrm{P}=0,005$ y pelágica versus $S$. californicus, $\mathrm{P}=0,012 ; \alpha=0,05)$. Por otra parte, el biovolumen del fitoplancton de red mostró diferencias significativas entre zonas pero no entre fechas, sin observarse interacción entre los factores. La zona pelágica y la zona colonizada por $S$. californicus difirieron significativamente entre sí (prueba de StudentNewman-Keuls, pelágica versus S. californicus, $\mathrm{P}=0,043 ; \alpha=0,05)$. Los valores de abundancia total fitoplanctónica registrados durante el período muestreado oscilaron entre 2098 cél $\mathrm{mL}^{-1}$, en la superficie de la E4 en

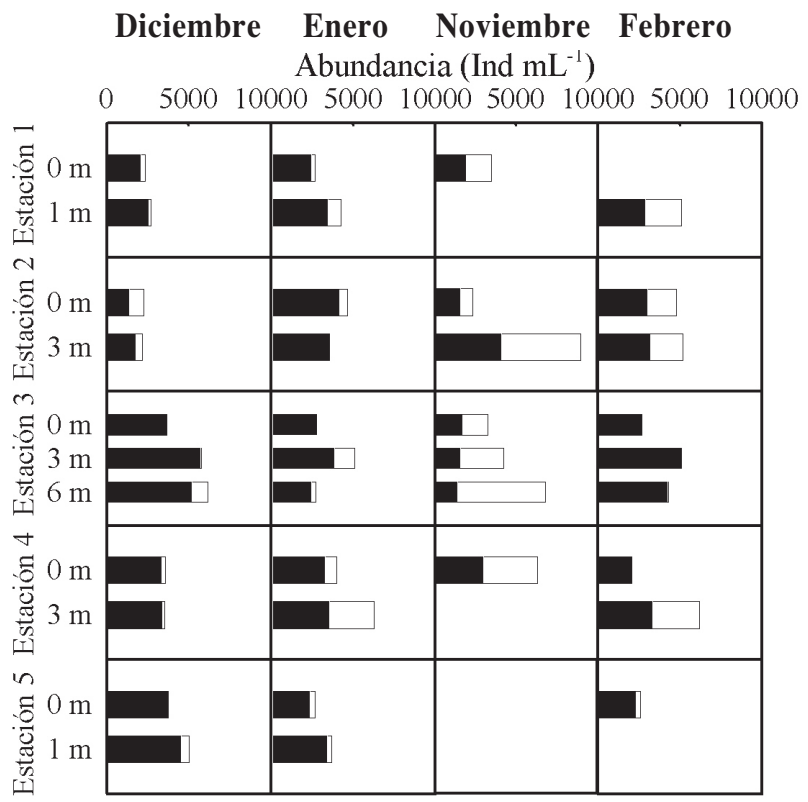

Diciembre Enero Noviembre Febrero

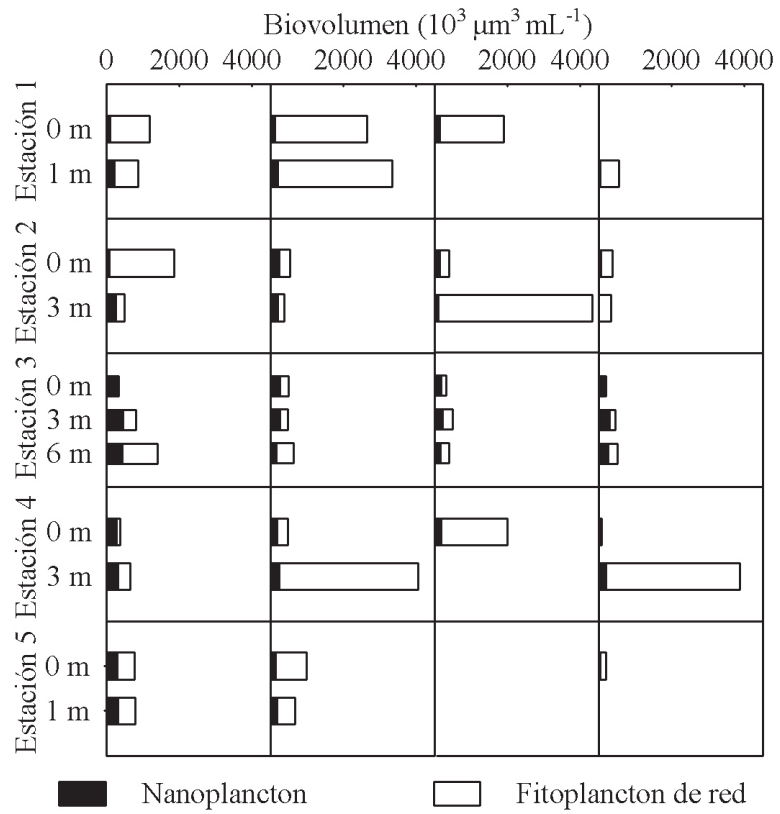

Fig. 2: Abundancia (A) y Biovolumen (B) de nanoplancton y fitoplancton de red en las diferentes estaciones de muestreo.

Nanoplankton and net phytoplankton abundances (A) and biovolume (B) at the sampling stations. 
febrero, y 8.973 células $\mathrm{mL}^{-1}$, a los $3 \mathrm{~m}$ de la E2 en noviembre. Sin embargo, las diferencias estadísticas entre zonas y fechas resultaron no significativas. La fracción nanoplanctónica estuvo dominada por un grupo de pequeños nanoflagelados constituido mayoritariamente por Chrysochromulina parva Lackey, Rhodomonas lacustris Pascher et Ruttner y Ochromonas sp. Por su parte, el fitoplancton de red estuvo constituido por cianofitas, clorofitas y dinofitas en la zona pelágica, mientras que en la zona litoral predominaron las diatomeas. Un caso particular lo representa el mes de noviembre en el que la cianofita colonial Coelosphaerium kuetzingiarum Näg. dominó en todas las estaciones y niveles de muestreo.

Los tecamebianos incluyeron 14 especies observándose un máximo de 11 en la E4 en noviembre y febrero (Tabla 3 ). Si bien las tecamebas se observaron mayormente en la zona litoral, se registró la presencia de los géneros Arcella y Difflugia en la estación pelágica, aunque con baja abundancia $(<3$ individuos $\left.\mathrm{L}^{-1}\right)$. Centropyxis aculeata, Arcella discoides, Trinema enchelys y Difflugia piriformis estuvieron presentes en todos los muestreos mientras que Euglypha ciliata, Euglypha strigosa y Lesquereusia spiralis fueron registradas solo en febrero y Trinema enchelys y Cyphoderia ampulla solo en noviembre y febrero. Se observaron diferencias en la abundancia entre las distintas estaciones y entre los distintos muestreos (Fig. 3). La mayor abundancia se observó en el nivel superficial de las estaciones litorales situadas en el sector norte del lago (E1 y E2) donde hay mayor densidad de macrófitas. Enero fue el período con los valores de abundancia más bajos (entre 1 y 16 individuos $\mathrm{L}^{-1}$ ), mientras que en noviembre, en coincidencia con el aumento de la cianofita Coelosphaerium, se registraron los valores máximos de abundancia (entre 112 y 725 individuos $\mathrm{L}^{-1}$ ).

Resultados de ANOVA de dos vías comparando biovolumen del nanoplancton, biovolumen del fitoplancton de red y Abundancia del Fitoplancton, en las diferentes estaciones y fechas de muestreo

Results of two-way ANOVA comparing Nanoplankton biovolume, net phytoplankton biovolume and phytoplankton abundance at the different sampling stations and dates

\begin{tabular}{|c|c|c|c|c|}
\hline Variable & Fuente de variación & Grados de libertad & Valor de F & Valor de $\mathrm{P}$ \\
\hline \multirow{5}{*}{ Biovolumen de nanoplancton } & Fecha de muestreo & 3 & 8,925 & $<0,001$ \\
\hline & Zona del lago & 2 & 6,49 & 0,005 \\
\hline & Interacción & 6 & 1,532 & 0,207 \\
\hline & Residual & 26 & & \\
\hline & Total & 37 & & \\
\hline \multirow[t]{5}{*}{ Biovolumen de fitoplancton de red } & Fecha de muestreo & 3 & 1,244 & 0,314 \\
\hline & Zona del lago & 2 & 3,466 & 0,046 \\
\hline & Interacción & 6 & 0,285 & 0,939 \\
\hline & Residual & 26 & & \\
\hline & Total & 37 & & \\
\hline \multirow[t]{5}{*}{ Abundancia del fitoplancton } & Fecha de muestreo & 3 & 0,485 & 0,696 \\
\hline & Zona del lago & 2 & 1,069 & 0,358 \\
\hline & Interacción & 6 & 1,022 & 0,433 \\
\hline & Residual & 26 & & \\
\hline & Total & 37 & & \\
\hline
\end{tabular}


TABLA 3

Especies de tecamebas registradas en las diferentes estaciones de muestreo (1, 2, 3, 4 y 5)

Testate amoebae species registered at the different sampling stations (1, 2, 3, 4 and 5)

\begin{tabular}{|c|c|c|c|c|c|c|c|c|c|c|c|c|c|c|c|c|c|c|c|}
\hline \multirow[t]{2}{*}{ Especie } & \multicolumn{5}{|c|}{ Diciembre } & \multicolumn{5}{|c|}{ Enero } & \multicolumn{4}{|c|}{ Noviembre } & \multicolumn{3}{|c|}{ Diciembre } & \multirow[b]{2}{*}{4} & \multirow[b]{2}{*}{5} \\
\hline & 1 & 2 & 3 & 4 & 5 & 1 & 2 & 3 & 4 & 5 & 1 & 2 & 3 & 4 & 1 & 2 & 3 & & \\
\hline \multicolumn{20}{|l|}{ Familia Arcellinidae } \\
\hline Arcella discoides Ehr. & & + & & & + & + & + & & + & + & + & + & & + & + & + & & + & + \\
\hline A. gibbosa Penard & + & + & + & & + & + & + & + & + & + & + & + & & + & + & + & & + & + \\
\hline A. hemisphaerica Defl. & & & + & + & + & + & + & + & + & + & + & + & & + & + & + & & + & + \\
\hline \multicolumn{20}{|l|}{ Familia Centropyxidae } \\
\hline Centropyxis aculeata (Ehr.) & + & + & & + & + & + & + & & & + & + & + & & + & + & + & & + & + \\
\hline \multicolumn{20}{|l|}{ Familia Difflugiidae } \\
\hline Difflugia acuminata Ehr. & & & & + & & & & & & & & & & + & & & & & \\
\hline D. elegans Pen. & & + & & + & + & + & + & & & + & + & + & & + & + & + & & + & \\
\hline D. gramen Pen. & + & + & + & + & & + & + & & + & + & + & + & & + & + & + & & + & + \\
\hline D. piriformis Ehr. & + & + & & & + & + & + & & + & + & & + & & + & + & + & & + & + \\
\hline \multicolumn{20}{|l|}{ Familia Lesquereusiidae } \\
\hline Lesquereusia spiralis (Ehr.) & & & & & & & & & & & & & & & + & & & & \\
\hline \multicolumn{20}{|l|}{ Familia Euglyphidae } \\
\hline Euglypha ciliata & & & & & & & & & & & & & & & & & & + & \\
\hline E. acantophora (Ehr.) & + & + & & + & & & + & & & & + & + & + & + & + & + & & + & \\
\hline E. strigosa (Ehr.) & & & & & & & & & & & & & & & & + & & & \\
\hline \multicolumn{20}{|l|}{ Familia Trinematidae } \\
\hline Trinema enchelys (Ehr.) & & & & & & & & & & & + & + & & + & + & + & & + & + \\
\hline \multicolumn{20}{|l|}{ Familia Cyphoderiidae } \\
\hline Cyphoderia ampulla (Ehr.) & & & & & & & & & & & + & + & & + & & & & + & \\
\hline Número de especies & 5 & 7 & 3 & 6 & 6 & 7 & 8 & 2 & 5 & 7 & 9 & 10 & 1 & 11 & 10 & 10 & 0 & 11 & 7 \\
\hline
\end{tabular}

Se registraron en total 32 especies de rotíferos siendo la familia Lecanidae la que presentó mayor número de especies, seguida por las familias Synchaetidae y Trichocercidae (Tabla 4). El estudio de la distribución de las distintas especies mostró patrones particulares (Fig. 4). Collotheca mutabilis, Keratella cochlearis, Lecane (M) lunaris, Polyarthra vulgaris y Synchaeta sp. estuvieron presentes en todas las ocasiones de muestreo y mostraron una mayor ubicuidad en el lago (Fig. 4, Tabla 4). Conochilus unicornis y Pompholix sulcata solo fueron registrados en la estación pelágica en bajas abundancias. Los géneros Trichocerca, Euchlanis, la mayor parte de Lecane y Monommata; y las especies Trichotria tetractis, Macrochaetus altamirai, Mytilina ventralis $\mathrm{y}$
Lepadella ovalis, se observaron en casi todas las estaciones litorales con abundancias muy bajas. La mayor riqueza específica se registró en las estaciones litorales siendo el valor máximo de 21 especies para las E2 y E4 en febrero. Por su parte, la estación pelágica fue la que presentó menor número de especies (entre siete y 10 especies). Por otra parte, la abundancia de individuos no mostró diferencias significativas entre las diferentes zonas; sin embargo, sí se observaron entre las fechas de muestreo $(\alpha=0,05)$ (Tabla 5). La menor abundancia total fue 17 individuos $\mathrm{L}^{-1}$ en la E2 en diciembre, mientras que el valor máximo se registró en enero y fue 414 individuos $\mathrm{L}^{-1}$ en la E3. Las especies de Synchaeta fueron muy abundantes en todas las estaciones y en la 
mayor parte de los muestreos, particularmente en el mes de noviembre, donde se registró el valor máximo en la E3 (332 individuos $\mathrm{L}^{-1}$ ). También se observaron valores elevados de abundancia para Polyarthra vulgaris, que alcanzó un máximo de 305 individuos $\mathrm{L}^{-1}$ en la E3 en el muestreo de enero. Las especies más abundantes en las estaciones litorales fueron Lecane spp (82 individuos $\left.\mathrm{L}^{-1}\right)$, en la $\mathrm{E} 1$ en diciembre, $P$. vulgaris en enero (100 individuos $\mathrm{L}^{-1}$ ) y en febrero (93 individuos $\mathrm{L}^{-1}$ ) y Synchaeta en noviembre (256 individuos $\mathrm{L}^{-1}$ ) (Fig. 4).

Al analizar la diversidad específica de tecamebianos y rotíferos, se observa que la estación pelágica presentó valores más bajos que las estaciones de muestreo colonizadas por macrófitas (Fig. 4). Para ambos grupos se observaron diferencias significativas entre las zonas del lago (Tabla 6). Además el análisis señaló diferencias entre la zona pelágica y cada macrófita (Tecamebas: prueba a posteriori de Student-Newman-Keuls, pelágica versus $P$. linguatus, $\mathrm{P}<0,001$ y pelágica versus $\mathrm{S}$. californicus, $\mathrm{P}<0,001, \alpha=0,05$; rotíferos: prueba a posteriori de Student-Newman-Keuls, pelágica versus $P$. linguatus, $\mathrm{P}=0,014 \mathrm{y}$ pelágica versus $S$. californicus, $\mathrm{P}=0,009 ; \mathrm{a}=$ $0,05)$. Sin embargo, no se hallaron diferencias entre las macrófitas (Tecamebas: prueba a posteriori de Student-Newman-Keuls, P. linguatus versus $\mathrm{S}$. californicus, $\mathrm{P}=0,193, \alpha=$ 0,05 ; rotíferos prueba a posteriori de StudentNewman-Keuls, $P$. linguatus versus $S$. californicus, $\mathrm{P}=0,323 ; \alpha=0,05)$.

En el ACC los primeros dos ejes explicaron el 59,6\% de la varianza (eje 1:39,3\%; eje 2:20,3\%) (Fig. 6). La prueba de permutaciones de Monte Carlo mostró que las abundancias del nanoplancton y el fitoplancton de red estuvieron significativamente correlacionados con los dos ejes $(\mathrm{P}<0,005)$. El primer eje estuvo definido por la abundancia de fitoplancton de red (coeficiente de correlación: $r=0,86)$, mientras que el segundo eje estuvo correlacionado con la abundancia del
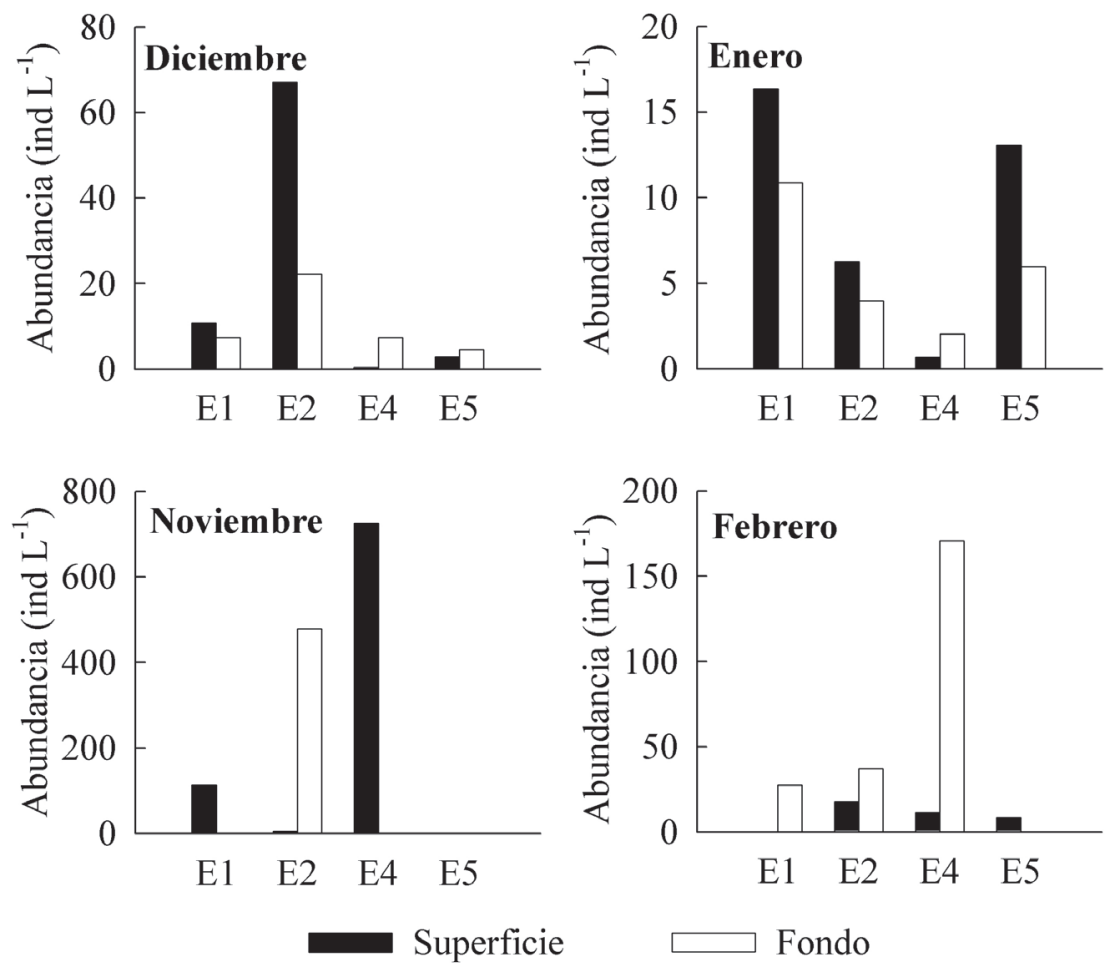

Fig. 3: Abundancia de tecamebianos en las E1, E2, E4 y E5. Los valores correspondientes a la E3 no se presentan debido a que las abundancias fueron mínimas $\left(0-2\right.$ ind $\left.\mathrm{L}^{-1}\right)$.

Testate amoebae abundances in E1, E2, E4 and E5. Values of the E3 are not shown because of their low abundances (0-2 ind $\mathrm{L}^{-1}$ ). 
TABLA 4

Especies de rotíferos registradas en las diferentes estaciones de muestreo (1, 2, 3, 4 y 5)

Rotifer species registered at the different sampling stations (1, 2, 3, 4 and 5)

\begin{tabular}{|c|c|c|c|c|c|c|c|c|c|c|c|c|c|c|c|c|c|c|c|}
\hline \multirow[t]{2}{*}{ Especie } & \multicolumn{5}{|c|}{ Diciembre } & \multicolumn{5}{|c|}{ Enero } & \multicolumn{4}{|c|}{ Noviembre } & \multicolumn{5}{|c|}{ Diciembre } \\
\hline & 1 & 2 & 3 & 4 & 5 & 1 & 2 & 3 & 4 & 5 & 1 & 2 & 3 & 4 & 1 & 2 & 3 & 4 & 5 \\
\hline
\end{tabular}

Orden Bdelloidea

Bdelloideo 1

Bdelloideo 2

Bdelloideo 3

Bdelloideo 4

Bdelloideo 5

Familia Collothecidae

Collotheca mutabilis (Hudson)

Familia Conochilidae

Conochilus unicornis Rousselet

Familia Testudinellidae

Pompholix sulcata (Hudson)

Testudinella patina (Herman)

Familia Brachionidae

Keratella cochlearis (Gosse)

Familia Colurellidae

Colurella uncinata (OFM)

Lepadella ovalis (OFM)

Familia Euchlanidae

Euchlanis dilatata Ehr.

Es triquetra Ehr.

Familia Gastropodidae Gastropus stylifer Imhof

Familia Lecanidae

Lecane bulla Gosse

L. lunaris (Ehr.)

L. closterocerca (Schmarda)

L. curvicornis (Murray)

L. stichaea Harring

L. flexilis (Gosse)

Lecane sp.

Familia Mytilinidae

Mytilina ventralis (Ehr.)

Familia Notommatidae

Monommata sp.

Familia Synchaetidae Polyarthra vulgaris Carlin

Synchaeta pectinata Ehr.

Synchaeta sp.

Familia Trichocercidae

Trichocerca longiseta (Shrank)

T. lophoessa (Gosse)

T. porcellus (Gosse)

Familia Trichotriidae

Macrochaetus sp.

Trichotria tetractis (Ehr.)

Número de especies
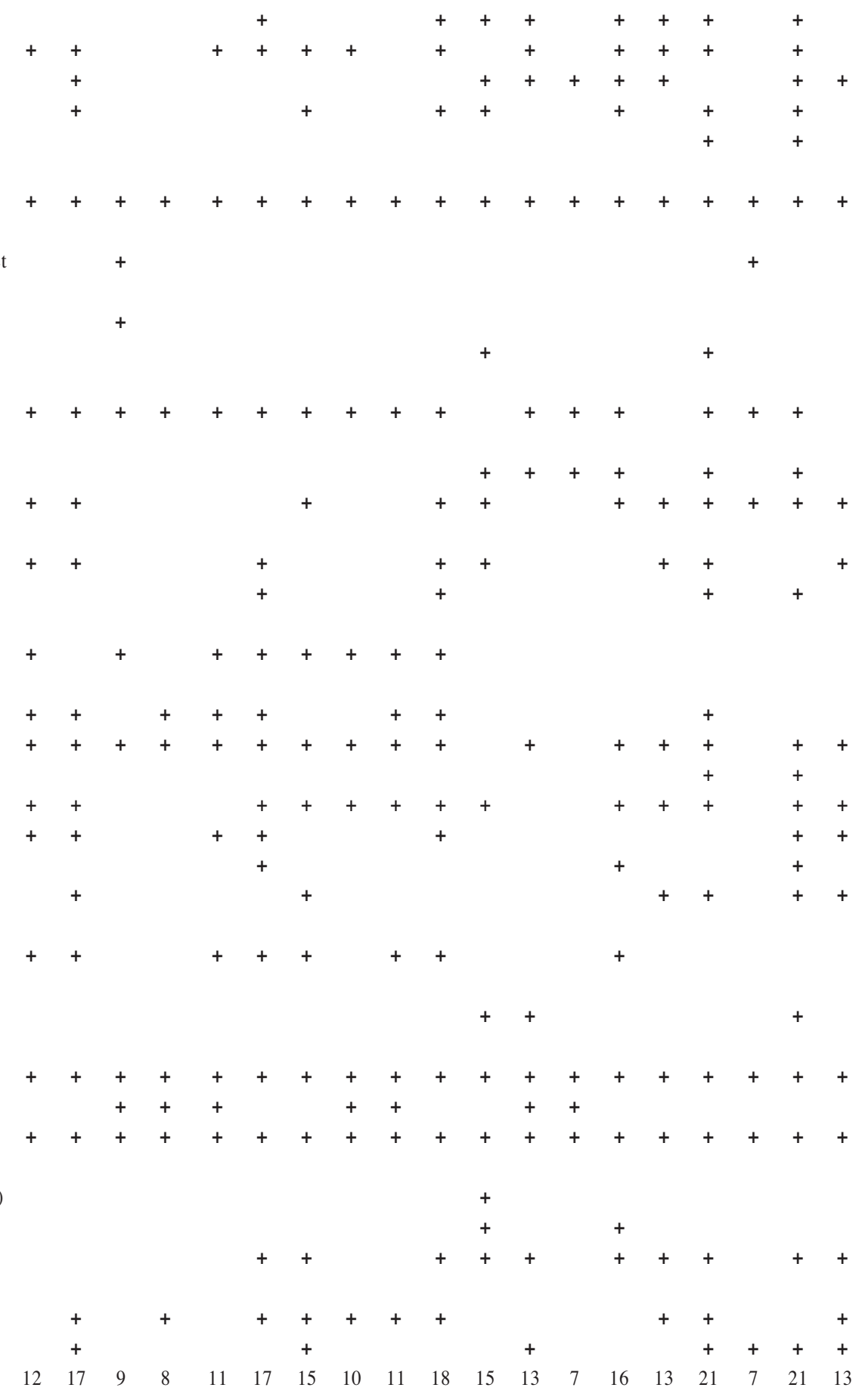


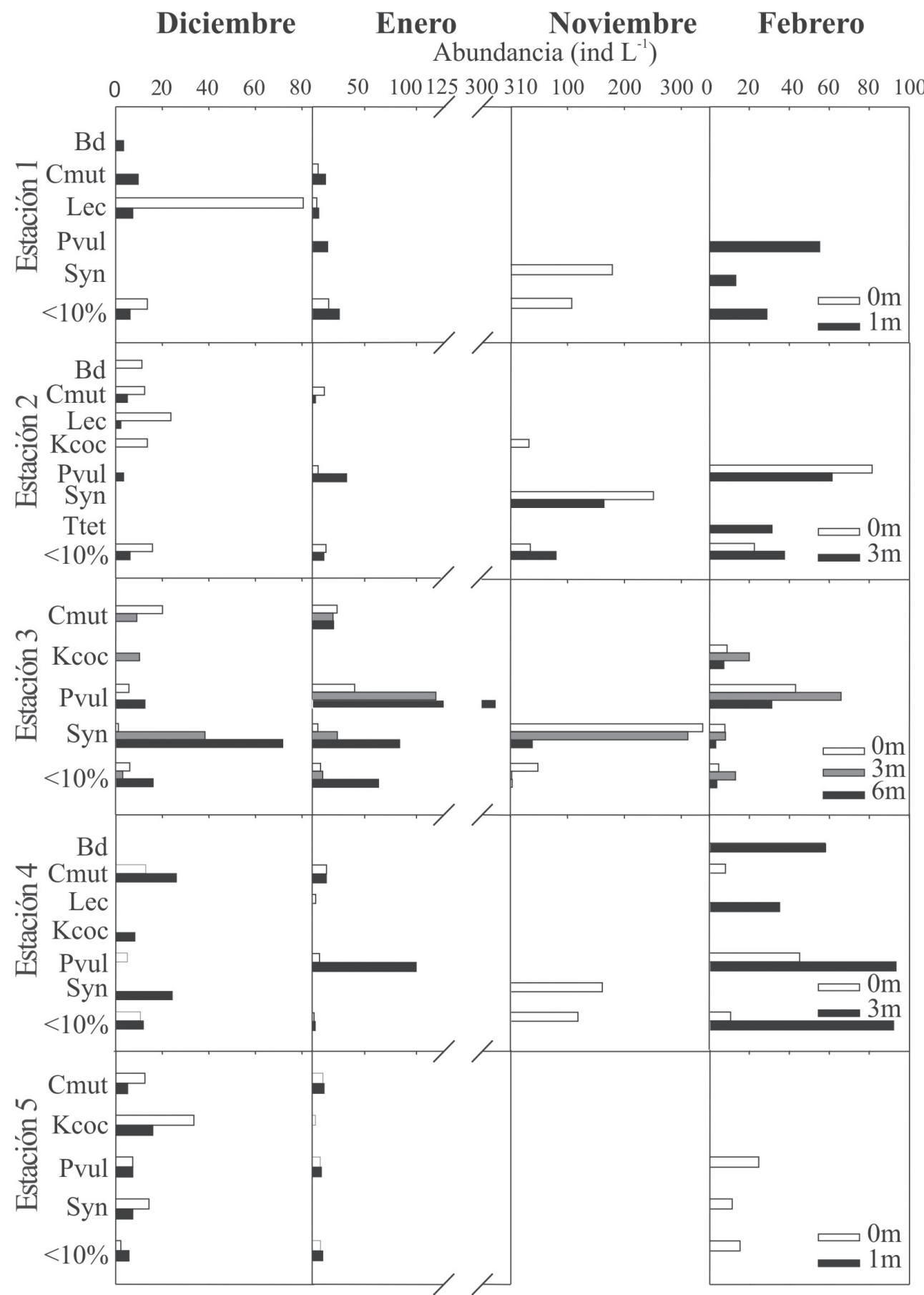

Fig. 4: Abundancia de las especies de rotíferos con frecuencia relativa mayor al $10 \%$. Referencias: Bd: Bdelloideos; Cmut: Collotheca mutabilis; Lec: Lecane spp; Kcoc: Keratella cochlearis; Pvul: Polyarthra vulgaris; Syn: Synchaeta spp; Ttet: Trichotria tetractis; < $10 \%$ : especies con frecuencia relativa menor al $10 \%$.

Rotifer abundances with relative frequency higher than $10 \%$. Reference: Bd: Bdelloideos; Cmut: Collotheca mutabilis; Lec: Lecane spp; Kcoc: Keratella cochlearis; Pvul: Polyarthra vulgaris; Syn: Synchaeta spp; Ttet: Trichotria tetractis; $<10 \%$ : species with relative frequency lower than $10 \%$. 
TABLA 5

Resultados de ANOVA de dos vías comparando abundancia de rotíferos en las diferentes estaciones y fechas de muestreo

Results of two-way ANOVA comparing rotifer abundance at the different sampling stations and dates

\begin{tabular}{lccc}
\hline Fuente de variación & Grados de libertad & Valor de F & Valor de P \\
\hline Fecha & 3 & 10,207 & $<0,001$ \\
Zona & 2 & 0,482 & 0,623 \\
Interacción & 6 & 0,675 & 0,671 \\
Residual & 26 & & \\
Total & 37 & & \\
\hline
\end{tabular}

TABLA 6

Resultados de ANOVA de dos vías comparando diversidad de rotíferos y tecamebas en las diferentes estaciones y fechas de muestreo

Results of two-way ANOVA comparing rotifer and testate amoebae diversity at the different sampling stations and dates

\begin{tabular}{|c|c|c|c|c|}
\hline Variable & Fuente de variación & Grados de libertad & Valor de F & Valor de $\mathrm{P}$ \\
\hline \multirow[t]{5}{*}{ Diversidad de rotíferos } & Fecha de muestreo & 3 & 1,085 & 0,373 \\
\hline & Zona del lago & 2 & 19,063 & $<0,001$ \\
\hline & Interacción & 6 & 2,381 & 0,057 \\
\hline & Residual & 26 & & \\
\hline & Total & 37 & & \\
\hline \multirow[t]{5}{*}{ Diversidad de tecamebas } & Fecha de muestreo & 3 & 1,837 & 0,179 \\
\hline & Zona del lago & 2 & 14,452 & $<0,001$ \\
\hline & Interacción & 6 & 0,862 & 0,542 \\
\hline & Residual & 17 & & \\
\hline & Total & 28 & & \\
\hline
\end{tabular}

nanoplancton (coeficiente de correlación: $\mathrm{r}=$ $0,74)$. Las especies pelágicas de rotíferos Keratella cochlearis, Synchaeta pectinata, Synchaeta sp., Polyarthra vulgaris $y$ Collotheca mutabilis se ubicaron en el sector superior izquierdo de la figura asociadas al nanoplancton (Fig. 6). De la misma manera la mayor parte de las muestras pelágicas (E3), también se encontraron en el mismo sector relacionadas con una mayor abundancia de nanoplancton. Solamente las muestras pelágicas de noviembre mostraron mayor relación con el fitoplancton de red, situándose en la zona inferior de la figura, debido a su correlación negativa con la abundancia de nanoplancton. Estas muestras también se vieron asociadas a Synchaeta sp., la cual fue muy abundante durante el muestreo de noviembre.
Las especies litorales de rotíferos Monommata sp., T. tetractis y los bdelloideos se relacionaron con abundancias altas de fitoplancton de red. Con respecto a las tecamebas, las especies de Difflugia y Trynema se asociaron, junto con los rotíferos mencionados, al fitoplancton de red.

\section{DISCUSIÓN}

En este estudio, la mayor abundancia y riqueza específica de tecamebas fueron siempre registradas en la zona litoral; sin embargo, algunas especies de Arcella y Difflugia fueron observadas en la estación limnética. De manera coincidente Green (1994) señala que las tecamebas habitan mayoritariamente en las 

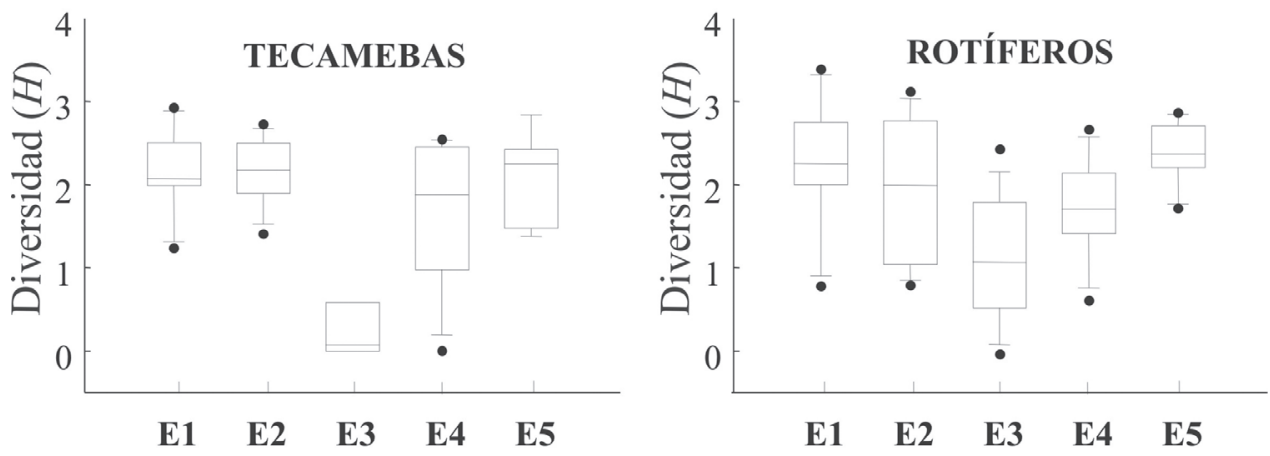

Fig. 5: Diversidad (H: Índice de Shannon y Weaver) de tecamebas y rotíferos en las diferentes estaciones de muestreo. Para cada estación de muestreo se observa la mediana, los cuartiles y los valores fuera de los percentiles del 10 y $90 \%$.

Testate amoebae and rotifer diversity (H: Shannon \& Weaver Index) at the sampling stations. Median, quartiles, and data outside 10th and 90th percentiles are indicated for each sampling station.

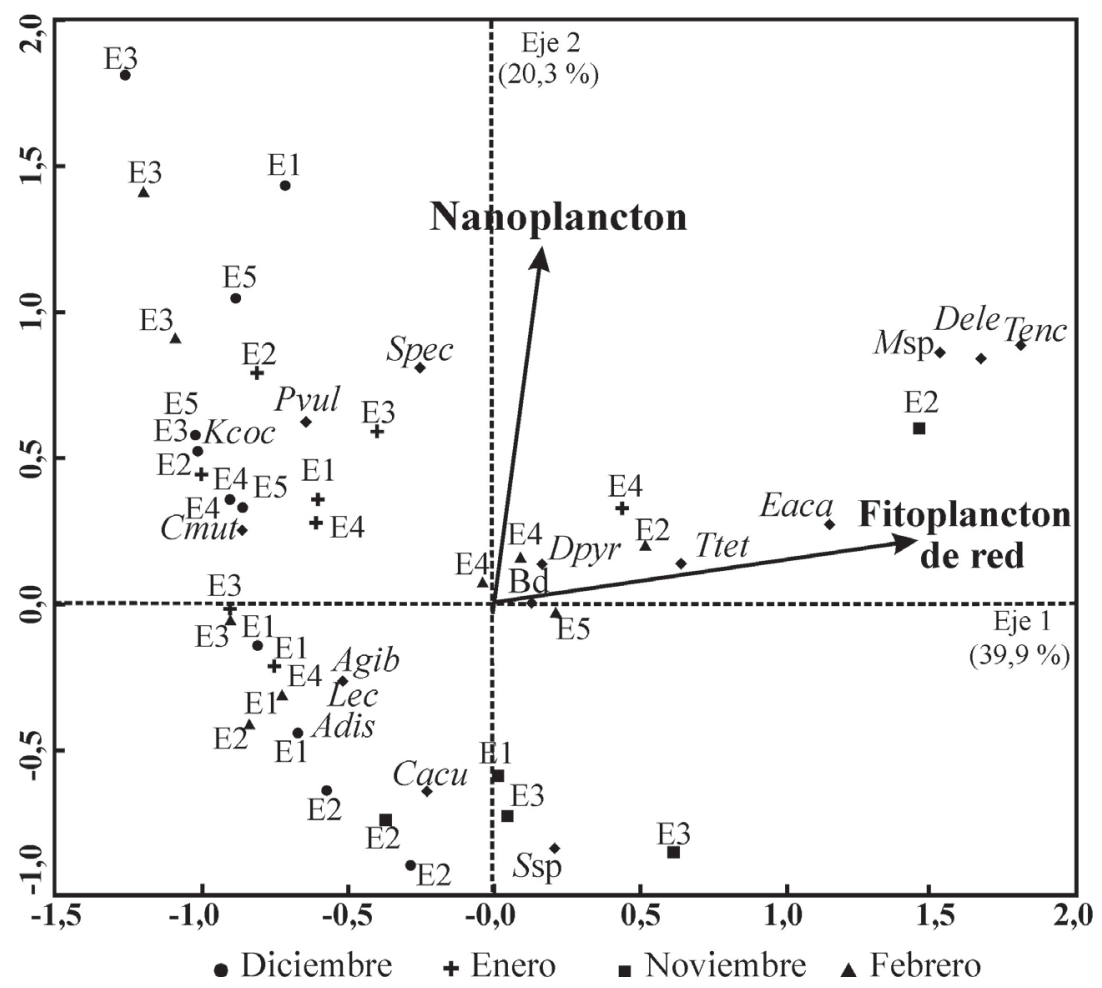

Fig. 6: ACC (Análisis de Correspondencia Canónica) de especies y estaciones de muestreo relativo a la abundancia de nanoplancton y fitoplancton de red (variables ambientales). Las variables ambientales se encuentran indicadas por flechas. Referencias: Bd: Bdelloideos; Cmut: Collotheca mutabilis; Lec: Lecane spp.; Kcoc: Keratella cochlearis; Pvul: Polyarthra vulgaris; Ssp: Synchaeta sp.; Spec: Synchaeta pectinata; Ttet: Trichotria tetractis; Msp: Monommata sp.; Tenc: Trynema enchelys; Dele: Difflugia elegans; Dpir: Difflugia piriformis; Eaca: Euplypha acantophora; Agib: Arcella gibbosa; Adis: Arcella discoides; Cacu: Centropyxis aculeata.

CCA (canonical correspondence analysis) of species and sampling stations in relation to the nanoplankton and net phytoplankton abundance (environmental variables). Reference: Bd: Bdelloideos; Cmut: Collotheca mutabilis; Lec: Lecane spp.; Kcoc: Keratella cochlearis; Pvul: Polyarthra vulgaris; Ssp: Synchaeta sp.; Spec: Synchaeta pectinata; Ttet: Trichotria tetractis; Msp: Monommata sp.; Tenc: Trynema enchelys; Dele: Difflugia elegans; Dpir: Difflugia piriformis; Agib: Arcella gibbosa; Adis: Arcella discoides; Cacu: Centropyxis aculeata. 
zonas vegetadas lacustres. Por otra parte, la presencia de tecamebas en la zona pelágica se relaciona con la capacidad de estos organismos para producir vacuolas de gas que les permiten moverse en la columna sin la ayuda de los movimientos del agua (Cicak et al. 1963, Grospietsch 1972, Ogden 1991). Las diferencias en cuanto a la abundancia y diversidad de tecamebianos entre las zonas litoral y pelágica del lago Escondido parecen estar vinculadas con la disponibilidad de alimento. Estudios previos han demostrado que el componente algal es muy importante en el régimen alimentario de los tecamebianos (Gilbert et al. 2000, Gilbert et al. 2003), aunque también pueden ingerir otros organismos aun aquellos inmóviles, senescentes o muertos (Gilbert et al. 2003). Por ejemplo, Modenutti \& Vucetich (1987) detectaron individuos de Arcella discoides, con diatomeas y clorofitas en el protoplasma y a Difflugia biconcava Ertl. predando sobre larvas nauplii. En la zona pelágica del lago Escondido se registró, en la mayoría de los muestreos, una escasa abundancia de células de mayor tamaño ( $>20$ $\mu \mathrm{m})$. Esta situación pudo haber condicionado la baja riqueza de tecamebianos en esta zona. Por el contrario, en la zona litoral la mayor abundancia de algas de mayor tamaño, en particular de diatomeas, constituiría una importante fuente de alimento que favorece la presencia de tecamebianos. Un estudio previo sobre las diatomeas del Lago Escondido (Bastidas-Navarro \& Díaz-Villanueva 2004) indicó la asociación de especies de Cymbella (C. fallaisensis y $C$. cymbiformis) en la zona litoral. Estas especies de gran tamaño $(\sim 60$ $\mu \mathrm{m})$ provienen del desprendimiento del epifiton y representarían al alimento característico de esta zona para las tecamebas.

Al analizar la distribución espacial de las especies de rotíferos presentes en el lago Escondido se observó que, a pesar de ser un lago pequeño, existen diferencias sustanciales entre las zonas pelágica y litoral. Estas diferencias se observan claramente en la diversidad específica, pero se hacen especialmente evidentes en la distribución diferencial de especies. En el lago Escondido se observaron cinco especies pelágicas: Keratella cochlearis, Polyarthra vulgaris, Synchaeta pectinata, Synchaeta sp. y Collotheca mutabilis. Cada una de estas especies presenta diferentes mástax, hábitos y estrategias de alimentación. Keratella cochlearis es una especie filtradora que se alimenta de células algales menores de $15 \mu \mathrm{m}$, especialmente pequeñas algas verdes, chrysomonas, cryptomonas y también detritus (Pourriot 1977, Ardnt \& Nixdorf 1991, Weisse \& Müller 1991). En el lago Escondido los nanoflagelados constituirían el principal alimento de Keratella cochlearis, de manera que es esperable que la abundancia de esta especie se encuentre directamente relacionada con la densidad de este grupo. Esto sucedió en diciembre de 2001, sin embargo, en enero de 2002 y febrero de 2003, hubo un descenso de $K$. cochlearis, a pesar que su fuente de alimento era abundante, concordando con un aumento considerable de la abundancia de Polyarthra vulgaris. Balseiro y Modenutti (1990) y Modenutti (1994) también observaron máximos de abundancia de $P$. vulgaris con una muy baja densidad de $K$. cochlearis, atribuyendo esta situación a competencia por alimento. Estas especies parecen competir por el mismo rango de tamaño de alimento, consumiendo principalmente nanoflagelados ya que la presencia de flagelo facilita la detección e ingestión de las células (Gilbert \& Bogdan 1984). Sin embargo, P. vulgaris presenta una estrategia de captura diferente ya que la presencia de mástax virgado y la morfología de la corona lleva a una captura más selectiva con respecto a la de $K$. cochlearis (Gilbert \& Bogdan 1984). Esta mayor selección del alimento podría favorecer el resultado de la interacción competitiva hacia $P$. vulgaris.

Por otra parte, algunas de las especies pelágicas registradas también podrían acceder a tamaños mayores de partículas. Las especies del género Synchaeta prefieren alimentarse de partículas de mayor tamaño $(40 \mu \mathrm{m})$ (Bogdan \& Gilbert 1987, Boon \& Shiel 1990) debido a que su mástax virgado les permite acceder con alta selectividad a las células succionando sus fluidos (Nogrady et al. 1993). Esto explica la relación entre el incremento en la abundancia de Synchaeta sp. y la cianófita colonial $C$. kuetzingianum registrado en noviembre en el lago Escondido. Otra especie pelágica que accede a partículas más grandes es $C$. mutabilis. Esta especie es raptora y se caracteriza por utilizar una estrategia de emboscada para capturar sus presas (Nogrady 
et al. 1993) siendo los ciliados su ítem alimentario preferido (Stemberger \& Gilbert 1987). Esta especie es muy común en lagos andinos someros y se relacionaría con una mayor abundancia en estos ambientes del pequeño ciliado Strobilidium sp. (Modenutti \& Diéguez 1996).

Debido a la gran cantidad de materia orgánica que precipita desde la columna de agua, la zona profunda de los lagos constituye una importante fuente de detritus (Wetzel 2001). Conochilus unicornis y Pompholix sulcata son especies que presentan mástax malleorramado adaptado para el molido y se alimentan del detrito y de las bacterias asociadas a este (Pourriot 1977). En el lago Escondido estas especie detritívoras se encontraron solamente en el fondo de la zona limnética, indicando la importancia del detritus para la dinámica de estas especies.

Con respecto a las especies litorales de rotíferos resulta evidente que su presencia se relaciona con un incremento en el tamaño de partícula del alimento. Las especies del género Trichocerca presentan mástax virgado que les permite succionar los fluidos de células algales (Stemberger 1979, Nogrady et al. 1993). Un estudio de la distribución de las diatomeas realizado en las diferentes zonas del lago Escondido mostró que los mayores valores de riqueza específica (30 especies) y de abundancia se observan en la zona litoral y las especies provienen en gran medida del epifiton (BastidasNavarro \& Diaz-Villanueva 2004). Por otra parte, las cianofitas filamentosas y coloniales, representadas por géneros tales como Coelosphaerium, Anabaena, Pseudoanabaena y Gloeocapsa, también adquieren importancia en la zona litoral. Como consecuencia de una mayor abundancia de estas células algales de mayor tamaño es esperable que las especies del género Trichocerca se restrinjan a la zona vegetada del lago. Por otro lado, se ha señalado que las especies de este género suelen también encontrarse en las muestras pelágicas y que la migración a la zona pelágica es más factible cuando la concentración de alimento es alta, es decir en cuerpos de agua eutróficos (Pejler \& Berzins 1993a). La ausencia de especies de Trichocerca en la zona pelágica del lago Escondido confirma su nivel de oligotrofía descrito previamente (Balseiro \& Modenutti 1990, Díaz \& Pedrozo 1993).
Otra especie litoral es Euchlanis dilatata que se alimenta de algas verdes y diatomeas (Pourriot 1977), así como de cianobacterias filamentosas (Gulati et al. 1993). Las observaciones ya mencionadas también explicarían la presencia restringida de $E$. dilatata en la zona litoral del lago Escondido, donde su fuente de alimento es más importante por la mayor abundancia de diatomeas y cianófitas.

También en la zona litoral una fuente importante de alimento es el detrito, cuya abundancia es sostenida principalmente por las macrófitas (Burks et al. 2002). Estas condiciones son propicias para el desarrollo de poblaciones de rotíferos detritívoros (José de Paggi 1993). En las estaciones litorales del lago Escondido las especies detritívoras observadas fueron: Lepadella ovalis, Colurella uncinata, Mytilina ventralis y Trichotria tetractis. Todas estas especies presentan mástax malleado y se alimentan de detrito y de sus bacterias asociadas (Pourriot 1977, Nogrady et al. 1993).

Si bien se observó una distribución diferencial de rotíferos entre las diferentes zonas, también se registró desplazamiento de determinadas especies entre las zonas litoral y pelágica. Las especies de Lecane, que se caracterizan por preferir los ambientes litorales, fueron también observadas en la zona limnética. Esto es debido a que no presentan gran dependencia del sustrato y son muy eurioicas (Pejler \& Berzins 1994). Por otro lado, Pejler \& Berzins (1993b) señalan que las especies de la familia Colurellidae también presentan alta movilidad y versatilidad y que, si bien son formas mayormente asociadas a sustratos, nadan libremente y a menudo aparecen en las muestras pelágicas. Por lo tanto, la presencia en la estación pelágica del lago Escondido de Colurella uncinata y Lepadella ovalis sería esperable. Por otra parte, Duggan et al. (2001) señalan que la capacidad de algunas especies pelágicas de habitar la zona litoral radica en que son formas planctónicas altamente móviles. En el lago Escondido dentro de este grupo se ubicaría a $P$. vulgaris y Synchaeta spp.

Las dos especies de macrófitas presentes en la zona litoral del lago Escondido, Schoenoplectus californicus y Potamogeton linguatus, difieren considerablemente en su arquitectura. Debido a que $P$. linguatus 
presenta mayor complejidad en su arquitectura, en comparación con $S$. californicus, podría existir una mayor diversidad de especies en la región de la macrófita sumergida. En este sentido, Ros (1983) observó una disminución en el número de especies relacionadas con $S$. californicus con respecto a la riqueza específica registrada en otras macrófitas. Sin embargo, en el lago Escondido no se observaron diferencias estadísticamente significativas en la diversidad de tecamebas y rotíferos entre las dos macrófitas. Asimismo, en un estudio sobre rotíferos del plancton y perifiton en ambientes lóticos pampásicos tampoco se observaron preferencias por una planta sustrato (Modenutti \& Claps 1988). Partiendo de la hipótesis inicial que la distribución de especies de tecamebas y rotíferos será distinta entre las zonas litoral y pelágica del lago Escondido, se puede concluir que dichas diferencias existen y son importantes. La distribución de los recursos alimentarios sería un factor determinante de dichas diferencias ya que ejercerían un papel preponderante en la distribución de especies en el lago Escondido.

\section{AGRADECIMIENTOS}

Este trabajo ha sido realizado en el marco de los proyectos CONICET PIP 6507 y FONCyT PICT 01-13395. M. Bastidas Navarro es becario CONICET y B. Modenutti es Investigador CONICET.

\section{LITERATURA CITADA}

ALEKPEROV I \& N SNEGOVAYA (2000) The fauna of testate amoebae (Rhizopoda, Testacea) in freshwater basins of Apsheron península. Protistology 1: 135-147.

ARDNT H \& B NIXDORF (1991) Spring clear-water phase un a eutrophic lake: control by herbivorous zooplankton enhanced by grazing on components of the microbial web. Verhandlungen Internationale Vereinigung Limnologie 24: 879-883.

BALSEIRO EG \& BE MODENUTTI (1990) Zooplankton dynamics of Lake Escondido (Rio Negro, Argentina), with special reference to a population of Boeckella gracilipes (Copepoda, Calanoidea). International Revue der gesamtem Hydrobiologie 75: 475-491.

BASTIDAS-NAVARRO MA \& V DÍAZ-VILLANUEVA (2004) Distribución espacial de las diatomeas en el fitoplancton de un lago somero andino patagónico. Boletín de la Sociedad Argentina de Botánica 39: 33-40.
BINI LM, LF VELHO \& FA LANSAC-TÔHA (2003) The effect of connectivity on the relationship between local and regional species richness of testate amoebae (protozoa, rhizopoda) in floodplain lagoons of the upper Paraná River, Brazil. Acta Oecologica 24: S145-S151.

BOGDAN KG \& JJ GILBERT (1987) Quantitative comparison of food niches in some freshwater zooplankton, a multi-tracer-cell approach. Oecologia 72: 331-340.

BOON PI \& J SHIEL (1990) Grazing on bacteria by zooplankton in Australian billabongs. Australian Journal of Marine \& Freshwater Research 41: 247 257.

BURKS RL, DM LODGE, E JEPPESEN (2002) Diel horizontal migration of zooplankton: costs and benefits of inhabiting the littoral. Freshwater Biology 47: 343-365.

CAMPOS H, W STEFFEN \& G AGŸERO (1988) Limnological study of Lake Llanquihue (Chile) Morphometry, physics, chemistry plankton and primary productivity. Archiv für Hydrobiologie (Suppl.) 81: 37-67.

CICAK A, JJA MCCAUGHLIN \& JB WITTENBERG (1963) Oxygen in the gas vacuole of the rhizopod protozoan Arcella. Nature 199: 983-985.

CYR H \& JA DOWNING (1988a) Empirical relationships of phytomacrofaunal abundance to plant biomass and macrophyte bed characteristics. Canadian Journal of Fisheries and Aquatic Sciences 45: 976 984.

CYR H \& JA DOWNING (1988b) The abundance of phytophilous invertebrates on different species of submersed macrophyte. Freshwater Biology 20: 365-374.

DÍAZ MM \& FL PEDROZO (1993) Seasonal succession of phytoplankton in a small Andean Patagonian lake (Rep. Argentina) and some consideration about the PEG Model. Archiv für Hydrobiologie 127: 167184.

DIEHL S (1992) Fish predation and benthic community structure-the role of omnivory and habitat complexity. Ecology 73: 1646-1661.

DUGGAN IC (2001) The ecology of periphytic rotifers. Hydrobiologia 446/447: 139-148.

DUGGAN IC, JD GREEN, K THOMPSON\& RJ SHIEL (2001) The influence of macrophytes on the spatial distribution of littoral rotifers. Freshwater Biology 46: 777-786.

DVORAK J (1970) Horizontal zonation of macrovegetation, water properties and macrofauna in a litoral stand of Glyceria aquatica (L.) Wahlb in a pond in south Bohemia. Hydrobiologia 35: 17-30.

DVORAK J \& DPH BEST (1982) Macro-invertebrate communities associated with macrophytes of Lake Vechten: structural and functional relationships. Hydrobiologia 95: 115-126.

GILBERT JJ \& KG BOGDAN (1984) Rotifer grazing: in situ studies on selectivity and rates. En: Meyer DG \& JR Strickler (eds) Trophic interactions within aquatic ecosystems: 97-133. AAAS Selected Symposia Series, Washington, District of Columbia, USA

GILBERT D, C AMBLARD, G BOURDIER \& RJ SHIEL (2000) Le régime aliementaire des Thécamoebiens (Protista, Sarcodina). L'Année Biologique 38: 57-68.

GILBERT D, EAD MITCHELL, C AMBLARD \& AJ FRANCEZ (2003) Population Dynamics and Food Preference of the testate Amoeba nebela tincta major-bohemica-collaris complex (Protozoa) in a 
Sphagnum peatland. Acta Protozoologica 21: 99. 104.

GREEN J (1994) The temperate-tropical gradient of planktonic protozoa and rotifera. Hydrobiologia 272: 13-26.

GROSPIETSCH T (1972) Protozoa. Testacea und Heliozoa. En: Elster HJ \& W Ohle (eds) Das Zooplankton der Binnengewässer: 1-30. Band XXVI. 1. Teil. E. Schweizerbart'sche Verlagsbuchhandlung, Stuttgart, Deutschland

GULATI RD, J EJSMONT-KARABIN \& G POSTEMA (1993) Feeding in Euchlanis dilatata lucksiana Hauer on filamentous cyanobacteria and a prochlorophyte. Hydrobiologia 255/256: 269-274.

JOSÉ DE PAGGI S (1993) Composition and seasonality of planktonic rotifers in limnetic and littoral region of a floodplain lake (Paraná river system). Revista de Biología Tropical 26: 53-63.

LANSAC-TÔHA FA, C COSTA BONECKER, LF VELHO \& A FONSECA LIMA (1997) Composiçao e abundância da comunidade zooplanctonica. En: de Vazzoler AE, AA Abostinho \& N Segatti Hahn (eds) A planície de inundaçao do Alto Rio Paraná: 117-155. Editora da Universidade Estadual de Maringa, Maringa, Brasil

LEPS J \& P SMILAUER (2003) Multivariate analysis of ecological data usig CANOCO. University Press of Cambridge, Cambridge, United Kingdom. 269 pp.

MODENUTTI BE (1994) Spring-summer succession of planktonic rotifers in a south Andes lake. International Revue der gesamtem Hydrobiologie 79: 373-383.

MODENUTTI B, E BALSEIRO, C CALLIERI R BERTONI \& C QUEIMALIÑOS (2005) Effect of UV-B and different PAR intensities on the primary production of the mixotrophic planktonic ciliate Stentor araucanus. Limnology and Oceanography 50:864-871.

MODENUTTI BE, EG BALSEIRO \& CP QUEIMALIÑOS (2000) Ciliate community structure in two south Andes lakes: the effect of lake water on Ophrydium naumanni distribution. Aquatic Microbial Ecology 21: 299-307.

MODENUTTI BE, EG BALSEIRO, CP QUEIMALIÑOS, DA AÑÓN SUAREZ, MC DIÉGUEZ \& RJ ALBARIÑO (1998) Structure and dynamics of food web in Andean lakes. Lakes \& Reservoirs: Research and Management 3: 179-186

MODENUTTI BE \& MC CLAPS (1988) Monogononta rotifers from plankton and periphyton of pampasic lotic environments (Argentina). Limnologica (Berlin) 19: 167-175.

MODENUTTI BE \& MC DIÉGUEZ (1996) Dinámica poblacional de Collotheca mutabilis (Rotífera Monogononta) en dos lagos australes de diferentes régimen térmico. Revista Brasileira de Biologia 56: 403-408.

MODENUTTI BE \& MC VUCETICH (1987) Variación especial de los tecamebianos del zooplankton del arroyo Rodríguez (Provincia de Buenos Aires). Limnobios 2: 671-675.

MORRIS DP, HE ZAGARESE, CE WILLIAMSON , EG BALSEIRO, BR HARGREAVES, BE MODENUTTI, R. MOELLER \& C. P. QUEIMALIÑOS (1995) The attenuation of UV radiation in lakes and the role of dissolved organic carbon. Limnology and Oceanography 40: 1381-1391.

NOGRADY T, RL WALLACE \& TW SNELL (1993) Biology, ecology and systematics. SPB Academic Publishing, The Hague, Netherlands. 142 pp.
NUSCH EA (1980) Comparison of different methods for chlorophyll and phaeopigment determination. Archiv für Hidrobiologie Beihtrage Ergebnisse der Limnologie 14: 14-36.

OGDEN CG (1991) Gas vacuole and flotation in the testate amoeba Arcella discoides. Journal of Protozoology 38: 269-270.

PEJLER B (1995) Relation to habitat in rotifers. Hydrobiologia 313/314: 267-278.

PEJLER B \& B BERZINS (1993a) On the ecology of Trichocerca. Hydrobiologia 263: 55-59

PEJLER B \& B BERZINS (1993b) On the ecology of Colurellidae. Hydrobiologia 263: 61-64.

PEJLER B \& B BERZINS (1994) On the ecology of Lecane. Hydrobiologia 273: 77-80.

PEROTTI MG, MC DIÉGUEZ \& FG JARA (2005) Estado del conocimiento de humedales del norte patagónico (Argentina): aspectos relevantes e importancia para la conservación de la biodiversidad regional. Revista Chilena de Historia Natural 78: 723-737.

POURRIOT R (1977) Food and feeding habits of Rotifera. Archiv für Hidrobiologie Beihtrage Ergebnisse der Limnologie 8: 243-260.

ROOKE JB (1984) The invertebrates fauna of four macrophytes in a lotic system. Freshwater Biology 14: $507-513$

ROOKE JB (1986a) Macroinvertebrates associated with macrophytes and plastic imitations in the Eramosa river, Ontario, Canada. Archiv für Hydrobiologie 106: 307-325.

ROOKE JB (1986b) Seasonal aspects of the invertebrate fauna of three species of plants and rock surfaces in a small stream. Hydrobiologia 134: 81-87.

ROS PJ (1983) Dynamics o periphytic communities. En: Wetzel RG (ed) Periphyton in freshwater ecosystems: 5-10. B.V. Junk Publishers, The Hague, Netherlands.

SCHMID-ARAYA JM (1991) The effect of food concentration on the life histories of Brachionus plicatilis (O.F.M.) and Encentrum linnhei Scott. Archiv fur Hydrobiologie 121: 87-102.

SCHMID-ARAYA JM (1993) Spatial distribution and population dynamics of a benthic rotifer, Embata laticeps (Murray) (Rotifera, Bdelloidea) in the bed sediments of a gravel brook. Freshwater Biology 30: 395-408.

SCHWEIZER A (1997) From littoral to pelagial: comparing the distribution of phytoplankton and ciliated protozoa along a transect. Journal of Plankton Research 19: 829-848.

SHIEL RJ (1976) Association of Entomostraca with weedbed habitats in a Billabong of Goulburn river, Victoria. Australian Journal of Marine \& Freshwater Research 27: 533-549.

SOUTHWOOD TRE (1978) Ecological methods. Chapman \& Hall, New York, New York, USA. 524 pp.

STEMBERGER RS (1979) A guide to rotifers to rotifers of the Laurentian great lakes. EPA Report, 600/479-021, Cincinnati, Ohio, USA.186 pp.

STEMBERGER RS \& JJ GILBERT (1987) Defenses planktonic rotifers against predators. En: Kerfoot WC \& A Sih (eds) Predation: 227-239. University Press of New England, Hanover, USA. 381pp.

SUN J \& DJ LIU (2003) Geometric models for calculating cell biovolume and surface area for phytoplankton. Journal of Plankton Research 25: 1331-1346.

TALBOT JM \& JC WARD (1987) Macroinvertebrates associated with aquatic macrophytes in Lake Alexandrina, New Zealand. New Zealand Journal of Marine and Freshwater Research 21: 199-213. 
TER BRAAK JF \& P SMILAUER (2002) CANOCO reference manual and CanoDraw for Windows user's guide: software for canonical community ordination (version 4.5). Microcomputer Power, Ithaca, New York, USA. 500 pp.

THOMASSON K (1963) Araucarian lakes. Acta Phytogeographica Suecica 47: 1-139.

UNDERWOOD AJ (1997) Experiments in ecology. Cambridge University Press, Cambridge, Massachusetts, USA. 504 pp.
VITT DH \& S BAYLEY (1984) The vegetation and water chemistry of four oligotrophic basin mires in northwestern Ontario. Canadian Journal of Botany 62: $1485-1500$.

WEISSE T \& H MÜLLER (1991) The annual cycle of heterotrophic freshwater nanoflagellates: role of botton-up versus top-down control. Journal of Plankton Research 13: 167-185.

WETZEL RG (2001) Limnology. Academic Press, San Diego, California, USA. 1,006 pp

Editor Asociado: Horacio Zagarese

Recibido el 9 de noviembre de 2006; aceptado el 4 de enero de 2007 